Correction

\title{
Correction: Sano, M., et al. Microbial Screening Based on the Mizoroki-Heck Reaction Permits Exploration of Hydroxyhexylitaconic-Acid-Producing Fungi in Soils. Microorganisms 2020, 8, 648
}

\author{
Mei Sano ${ }^{1}$, Ryoki Yada ${ }^{1}$, Yusuke Nomura ${ }^{1}$, Takahiro Kusukawa ${ }^{2} \mathbb{D}$, Hiroshi Ando ${ }^{3}$, \\ Keiji Matsumoto $^{3}$, Kazuhito Wada ${ }^{3}$, Tomonari Tanaka ${ }^{1}\left(\mathbb{D}\right.$, Hitomi Ohara ${ }^{1}$ and Yuji Aso ${ }^{1, *(D)}$ \\ 1 Department of Biobased Materials Science, Kyoto Institute of Technology, Kyoto 606-8585, Japan; \\ d6861001@edu.kit.ac.jp (M.S.); m7661008@edu.kit.ac.jp (R.Y.); m8661011@edu.kit.ac.jp (Y.N.); \\ t-tanaka@kit.ac.jp (T.T.); ohara@kit.ac.jp (H.O.) \\ 2 Department of Chemistry and Materials Technology, Kyoto Institute of Technology, Kyoto 606-8585, Japan; \\ kusu@kit.ac.jp \\ 3 Corporate Research \& Business Division, Kaneka Corporation, Osaka 530-8288, Japan; \\ Hiroshi.Ando@kaneka.co.jp (H.A.); Keiji.Matsumoto@kaneka.co.jp (K.M.); \\ Kazuhito.Wada@kaneka.co.jp (K.W.) \\ * Correspondence: aso@kit.ac.jp; Tel.: +81-75-724-7694
}

The authors wish to make the following correction to this paper [1]: the compound names '8-hydroxyhexylitaxconic acid' and '9-hydroxyhexylitaconic acid' should be '9-hydroxyhexylitaxconic acid' and '10-hydroxyhexylitaconic acid', respectively, throughout the manuscript. For this correction, the text in the graphical abstract and the carbon numbering in Figure 4 should be changed. The authors would like to replace the original graphical abstract and Figure 4:

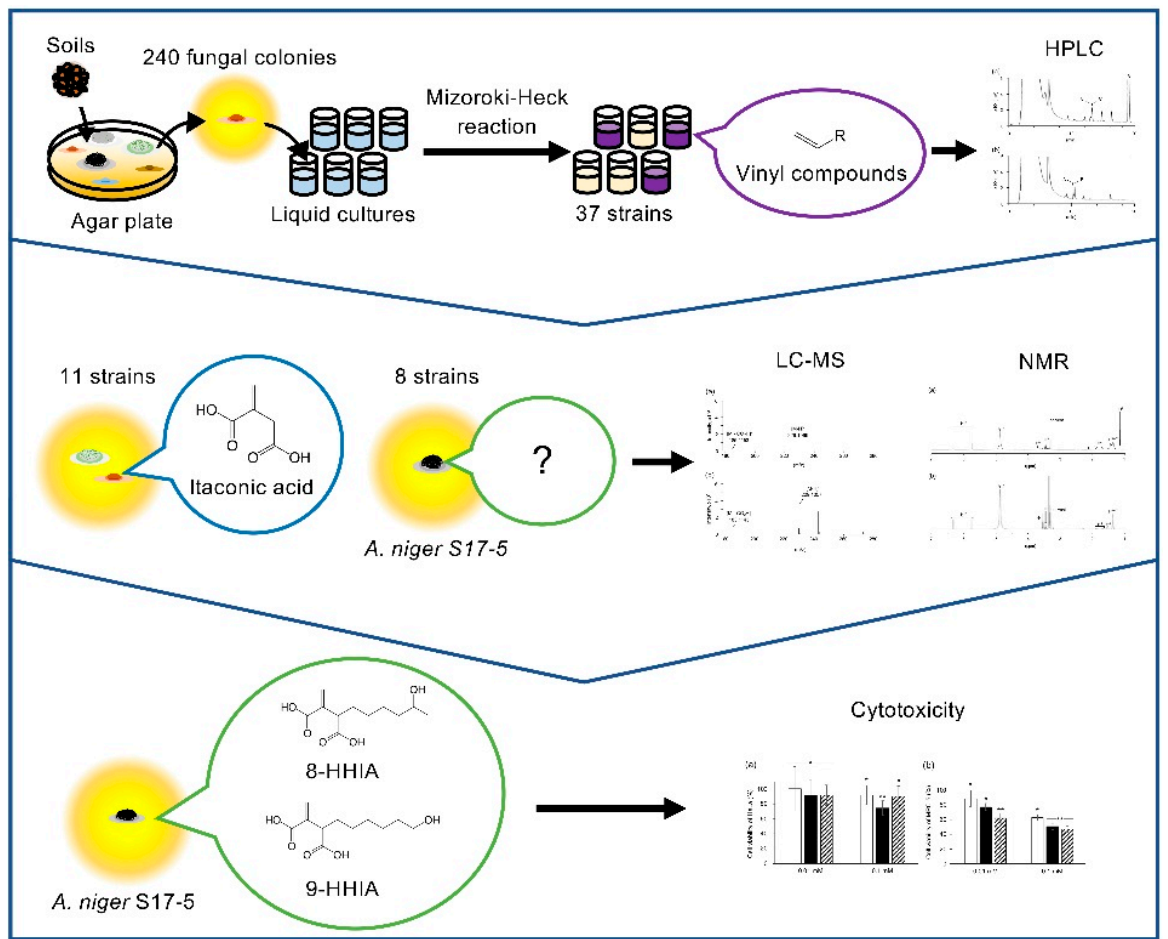


(a)

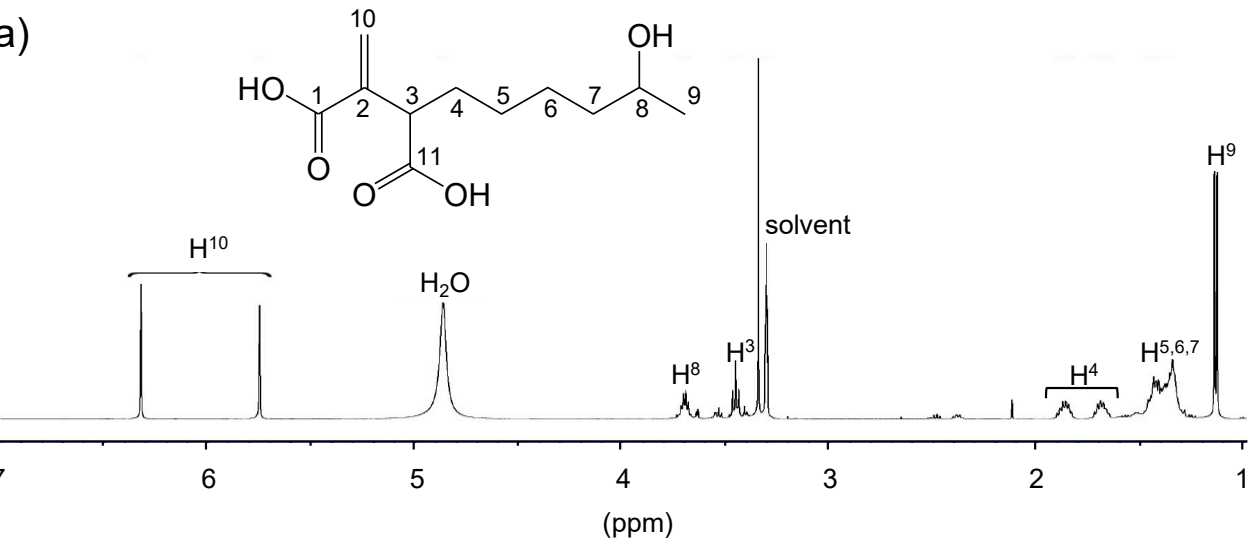

(b)

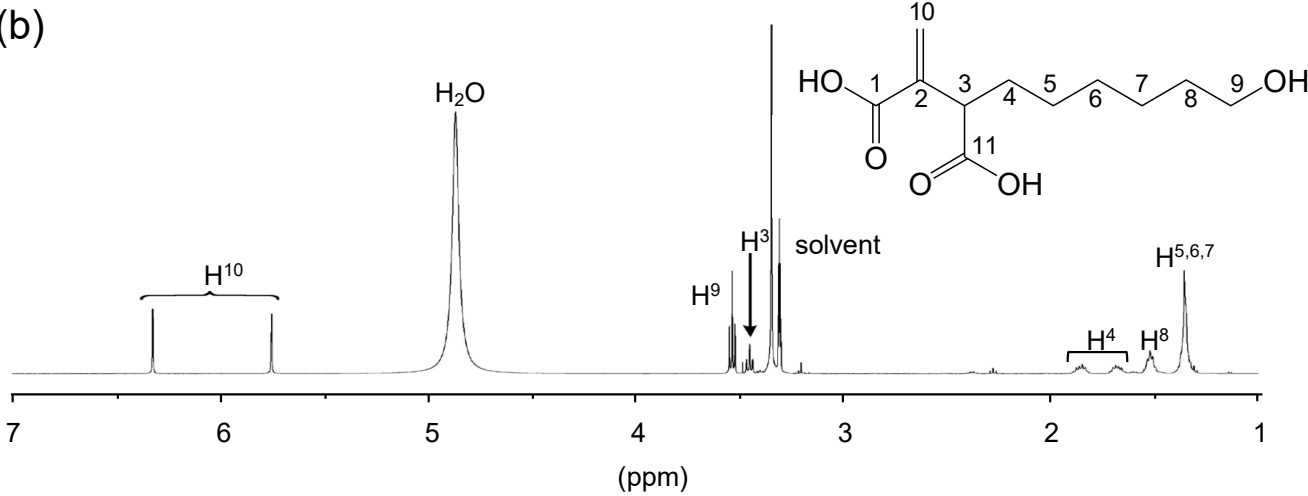

with new updated graphical abstract and Figure 4, respectively:

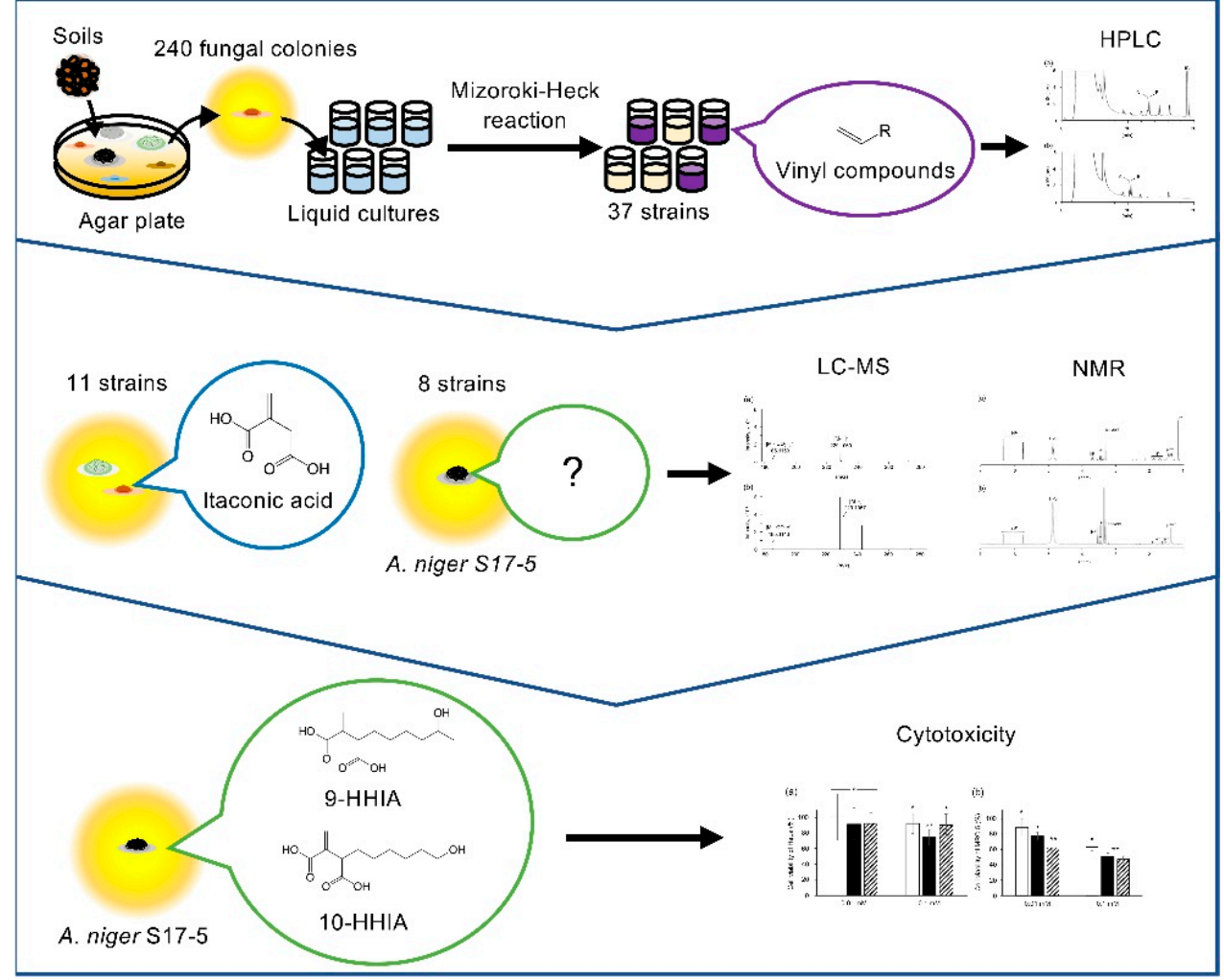



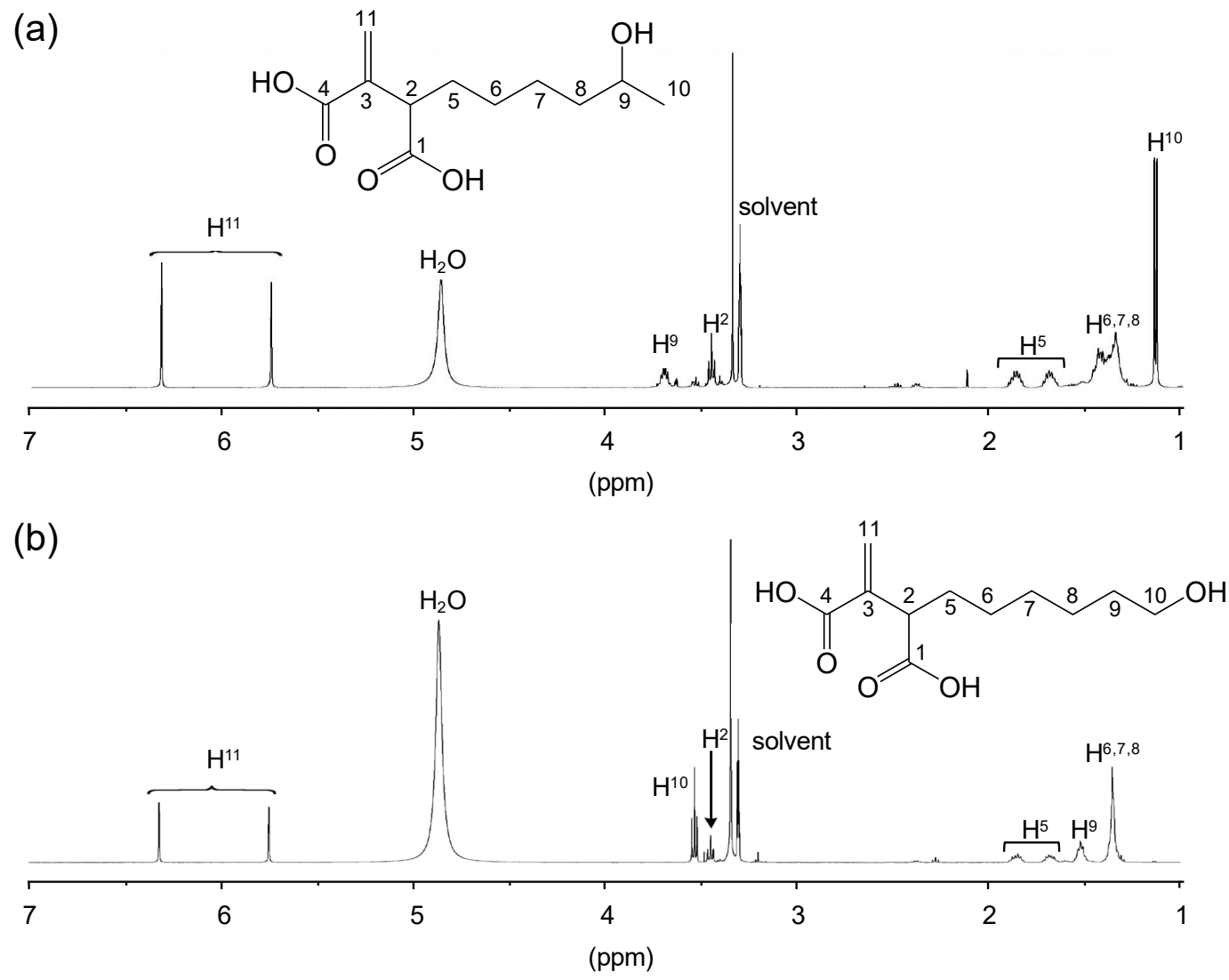

The manuscript will be updated, and the original will remain online on the article webpage, with a reference to this correction. The authors would like to apologize for any inconvenience caused to the readers by this change.

\section{Reference}

1. Sano, M.; Yada, R.; Nomura, Y.; Kusukawa, T.; Ando, H.; Matsumoto, K.; Wada, K.; Tanaka, T.; Ohara, H.; Aso, Y. Microbial screening based on the Mizoroki-Heck reaction permits exploration of hydroxyhexylitaconicacid-producing fungi in soils. Microorganisms 2020, 8, 648. [CrossRef] [PubMed]

(C) 2020 by the authors. Licensee MDPI, Basel, Switzerland. This article is an open access article distributed under the terms and conditions of the Creative Commons Attribution (CC BY) license (http://creativecommons.org/licenses/by/4.0/). 